\title{
Effects of whole-body cryotherapy on serum mediators of inflammation and serum muscle enzymes in athletes
}

\author{
Giuseppe Banfi ${ }^{\text {a,b }}$, Gianluca Melegati ${ }^{\text {a,c }}$, Alessandra Barassi ${ }^{\text {d }}$, Giada Dogliotti ${ }^{\text {e }}$, \\ Gianvico Melzi d'Eril ${ }^{\mathrm{d}}$, Benoit Dugué ${ }^{\mathrm{f}}$, Massimiliano M. Corsi ${ }^{\mathrm{a}, \mathrm{e}, *}$ \\ a Istituto Ortopedico R. Galeazzi, IRCCS, 20161 Milan, Italy \\ b Department of Health Technology, University of Milan, Milan, Italy \\ c Italian Rugby Federation, Italy \\ ${ }^{\mathrm{d}}$ Department of Medicine and Surgery, San Paolo Hospital, University of Milan, Milan, Italy \\ e Laboratory of Clinical Pathology, Institute of General Pathology, Medical Faculty, University of Milan, 20133 Milan, Italy \\ ${ }^{\mathrm{f}}$ Laboratoire des Adaptations Physiologiques aux Activités Physiques, Université de Poitiers, 86034 Poitiers Cedex, France
}

\section{A R T I C L E I N F O}

Article history:

Received 13 May 2008

Accepted 31 October 2008

Keyword:

Inflammation

Cryotherapy

Athletes

\begin{abstract}
A B S T R A C T
Whole-body cryotherapy (WBC) covers a wide range of therapeutic applications and consists of briefly exposing the body to extremely cold air. In sports medicine, WBC is used to improve recovery from muscle injury; however, empirical studies on its application to this area are lacking. To fill this gap, we compared changes in immunological parameters (C3, IgA, IgM, IgG, C-reactive protein, PGE2), cytokines (IL-2, IL-8, IL-10), adhesion molecules (sICAM-1), and muscle enzymes (creatine kinase [CK], lactate dehydrogenase [LAD]) before and after WBC in 10 top-level Italian National team rugby players. The subjects underwent five sessions on alternate days once daily for 1 week. During the study period, the training workload was the same as that of the previous weeks. Compared to baseline values, immunological parameters remained unchanged, while CK and LAD levels significantly decreased after treatment. No alterations in immunological function were observed but there is a decrease in proinflammatory cytokine/chemokine and an increase in anti-inflammatory cytokine.

As measured by changes in serum CK and LAD concentrations, and cytokines pathway, short-term cold air exposure was found to improve recovery from exercise-induced muscle injury and/or damage associated with intense physical training.
\end{abstract}

(c) 2008 Elsevier Ltd. All rights reserved.

\section{Introduction}

Whole-body cryotherapy (WBC) consists of brief exposure to extreme cold in a temperature-controlled chamber $\left(-110^{\circ} \mathrm{C}\right)$ (Westerlund et al., 2004). It is applied to relieve pain and inflammatory symptoms caused by numerous disorders, particularly those associated with rheumatic conditions, and is recommended for the treatment of arthritis, fibromyalgia and ankylosing spondylitis. In sports medicine, WBC has gained wider acceptance as a method to improve recovery from muscle injury; however, no controlled studies have been published so far.

WBC has been shown not to be deleterious to lung function (Smolander et al., 2006) or to decrease antioxidant capacity (Dugué et al., 2005) or propiomelanocortin-related hormones (Fricke et al., 1988). In a previous study, we demonstrated that

\footnotetext{
* Corresponding author at: Istituto Ortopedico R. Galeazzi, IRCCS, 20161 Milan, Italy.

E-mail address: mmcorsi@unimi.it (M.M. Corsi).
}

WBC does not enhance hematological values, as measured by hemoglobin concentration and counts of erythrocytes, reticulocytes, leukocytes, and platelets in peripheral blood (Banfi et al., 2008). Studies investigating the effects of cold exposure on immune function (Walsh and Whitham, 2006) found that lymphocyte, monocyte and tumor necrosis factor $\alpha$ levels were increased, whilst concentrations of interleukins IL-6, IL- $1 \beta$, and C-reactive protein (CRP) were unchanged after 6 weeks of cold water immersions (Jansky et al., 1996). Moreover, resting levels of IL-6, lymphocytes and monocytes were noted to be higher in subjects accustomed to winter swimming than in inexperienced individuals (Dugué and Leppänen, 2000). Contrary to popular belief, cold exposure can actually stimulate rather than depress immune function (Walsh and Whitham, 2006) In general, WBC does not appear to be harmful; indeed, it may be beneficial for athletes since prompt recovery from muscle injury is a primary concern for both athletes and sports physicians alike. Despite the wealth of literature on rehabilitation techniques, published data on WBC in rehabilitation programs are scarce. Studying the effects of WBC can have practical value not only for many physiological 
and clinical purposes, but also for determining clinical significance in the context of antidoping testing, since techniques that accelerate recovery may be classified as prohibited. Furthermore, post-WBC treatment changes in biochemical and hematological parameters could result outside the threshold range imposed by sports federations and official control agencies, with the athletes classified as being doped, or could be interpreted as an attempt to mask changes caused by illicit treatment different from WBC.

The aim of this study was to determine whether WBC had a positive effect on musculoskeletal metabolism, recovery from exertional muscle damage, and immune function. Biochemical and immunological markers were measured at baseline and after 1 week of WBC treatment (five once-daily sessions) in 10 male rugby players selected randomly from the Italian National team.

\section{Methods}

Ten male athletes (mean age $26 \pm 2.5$ years; mean body-mass index $27.5 \pm 2.3 \mathrm{~kg} / \mathrm{m}^{2}$ ) underwent once daily WBC treatment for 5 days (Monday-Wednesday, Friday, and Saturday) at the Olympic Rehabilitation Center of Spała (Poland). Wearing minimal clothing, the subjects were first exposed to very cold air $\left(30 \mathrm{~s}\right.$ at $\left.-60^{\circ} \mathrm{C}\right)$ then to extremely cold air $\left(2 \mathrm{~min}\right.$ at $\left.-110^{\circ} \mathrm{C}\right)$. They reported an improved sense of general well-being and no discomfort or disturbance.

During the study period, the subjects continued with their regular training. The workload was the same as in the previous 6 weeks. Training consisted of $3 \mathrm{~h}$ of daily exercises: $1 \mathrm{~h}$ of maximal training in the morning, followed by $1 \mathrm{~h}$ of submaximal effort, then $1 \mathrm{~h}$ of submaximal training in the afternoon, in addition to conditioning exercises.

No illnesses occurred during the study period. Diet was controlled and identical to that of the previous 6 weeks.

The 10 subjects were chosen randomly from the Italian National rugby team (30 athletes). All gave informed consent to the study protocol. Blood samples were drawn by vacutainer tubes at 8 a.m. on the first day of treatment (Monday) and then at the end of treatment on the following Monday. All subjects continued with the same training workload as that of the previous weeks. The time period from the last intense training session was the same for both blood drawings. The serum samples were separated within $3 \mathrm{~h}$ from drawing and stored at $-20^{\circ} \mathrm{C}$ until assayed. All biochemical parameters were measured on a Roche Modular (Roche, Basel, Switzerland), except for high-sensitivity CRP (hs-CRP), which was measured nefelometrically on a BN ProSpec analyzer (Behring, Marburg, Germany). PGE2, cytokines (IL-2, IL-8, IL-10) and sICAM-1 were measured with ELISA kits (R\&D Systems, Minneapolis, MN, USA) and read on an spectrophotometer (GDV, Milan, Italy).

Statistical analysis was performed using the paired $t$ test on a MedCalc program (Mariawerke, Belgium). Statistical significance was set at $p<0.05$.

\section{Results}

Table 1 compares pre- and post-treatment blood chemistry values. A slight but not significant increase in Ig and a slight but not significant decrease in CRP were noted. Lymphocyte and monocyte counts remained substantially unchanged $(p=$ n.s.): $44.7 \pm 8.2 \%$ versus $37.8 \pm 10.6 \%$ and $9.6 \pm 1.7 \%$ versus $9.6 \pm 3.5 \%$, respectively. $\mathrm{LAD}$ and $\mathrm{CK}$ both decreased significantly: $307.7 \pm 103.2 \mathrm{U} / \mathrm{L}$ versus $183.9 \pm 83.4 \mathrm{U} / \mathrm{L}$ and $362.3 \pm 34.3 \mathrm{U} / \mathrm{L}$ versus $318.4 \pm 28.6 \mathrm{U} / \mathrm{L}$, respectively. The standard deviation in
Table 1

Serum concentration of immunological markers and muscle enzymes before and after whole-body cryotherapy (WBC) in 10 top-level rugby players.

\begin{tabular}{lccl}
\hline & Before WBC & After WBC & $P$ value \\
\hline IgG $(\mathrm{mg} / \mathrm{dL})$ & $1262.4 \pm 196.5$ & $1286.3 \pm 186.4$ & n.s. \\
IgM $(\mathrm{mg} / \mathrm{dL})$ & $97.9 \pm 33.7$ & $100.5 \pm 35.1$ & n.s. \\
IgA $(\mathrm{mg} / \mathrm{dL})$ & $240.1 \pm 106.2$ & $250.3 \pm 116.3$ & n.s. \\
CRP $(\mathrm{mg} / \mathrm{dL})$ & $0.74 \pm 0.43$ & $0.62 \pm 0.38$ & n.s. \\
C3 $(\mathrm{U} / \mathrm{L})$ & $140.3 \pm 20.9$ & $142.5 \pm 21.6$ & n.s. \\
CK $(\mathrm{U} / \mathrm{L})$ & $307.7 \pm 103.2$ & $183.9 \pm 83.4$ & $<0.01$ \\
LAD $(\mathrm{U} / \mathrm{L})$ & $362.3 \pm 34.3$ & $318.4 \pm 28.6$ & $<0.01$ \\
PGE2 $(\mathrm{pg} / \mathrm{mL})$ & $1162.9 \pm 292.3$ & $351.5 \pm 179.9$ & $<0.0001$ \\
\hline
\end{tabular}

\pm values are means $\pm \mathrm{SD}$. $\mathrm{CRP}-\mathrm{C}$-reactive protein; $\mathrm{C} 3-\mathrm{C} 3$ proactivator; $\mathrm{CK}-$ creatine kinase; LAD_lactate dehydrogenase; PGE2-prostaglandin E2.

Table 2

Serum concentration of cytokines and adhesion molecule before and after wholebody cryotherapy (WBC) in 10 top-level rugby players.

\begin{tabular}{lccc}
\hline & Before WBC & After WBC & $P$ value \\
\hline IL-2 pg/mL & $11.37 \pm 5.91$ & $6.47 \pm 3.66$ & $<0.05$ \\
IL-8 pg/mL & $10.30 \pm 2.31$ & $8.18 \pm 2.11$ & $<0.05$ \\
IL-10 pg/mL & $41.15 \pm 3.07$ & $45.84 \pm 3.55$ & $<0.01$ \\
SICAM-1 ng/mL & $196.14 \pm 113.12$ & $74.68 \pm 44.11$ & $<0.01$ \\
\hline
\end{tabular}

IL-2_interleukin-2; IL-8_interleukin-8; IL-10_interleukin-10; sICAM-1—soluble intercellular adhesion molecule 1.

the post-treatment values decreased, indicating a greater uniformity of data.

The hs-CRP assay is a highly sensitive test for in measuring CRP levels in inflammation associated with rheumatoid arthritis and cardiac disease. The results of the hs-CRP test were similar to those obtained with conventional CRP testing. The mean hs-CRP before and after WBC was $0.88 \pm 0.40$ and $0.77 \pm 0.40 \mathrm{mg} / \mathrm{L}$, respectively ( $p=$ n.s.). Table 2 shows pro-inflammatory cytokines, prostacyclin, and adhesion molecule: a slight significant decrease of IL- $2(p<0.05)$ and IL-8 $(p<0.05)$, and a very significant increase of IL-10 $(p<0.05)$ were noted. PGE2 was significantly decreased $(p=<0.0001)$. Moreover also sICAM-1 decreased in a very significant way $(p<0.01)$ (Fig. 1$)$

\section{Discussion}

The use of methods other than passive recovery for improving recovery after intense training and competitions in sports is growing. In particular, in rugby, the use of cold water, possibly associated with active recovery (cycling), and the use of cold and hot water immersion are quite popular. WBC refers to brief exposure to very cold air for treating symptoms of various illnesses. In sports medicine, WBC is administered to improve recovery from muscular trauma. As specific studies are lacking, we measured immunological and muscular markers in 10 top-level rugby players of the Italian National team before and after a 1 -week course of daily sessions of WBC. The cold has also been shown to increased concentrations of anti-inflammatory cytokines in peripheral blood: it is suggested to have local and systemic anti-inflammatory effect.

We chose to compare changes in immunoglobulin and CRP levels because they are reliable indicators of acute or chronic infection and/or inflammation. Widely available in clinical laboratories, these markers are routinely and easily evaluated in the general population and in athletes. Lymphocyte and monocyte counts and plasma IL-6 concentrations are known to be higher in experienced than in inexperienced winter swimmers, probably 
a
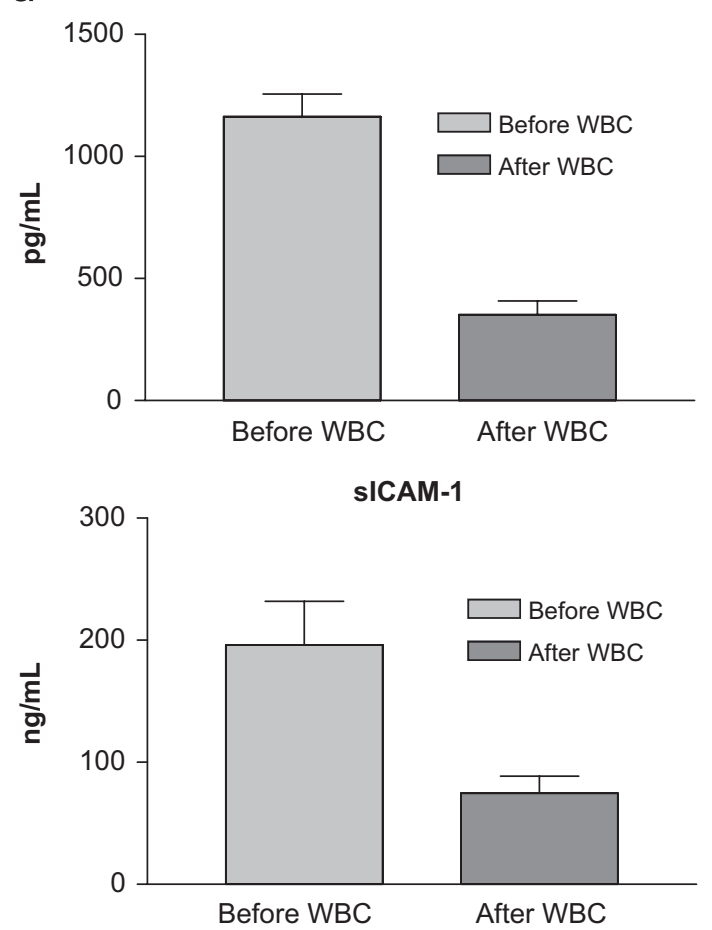

C

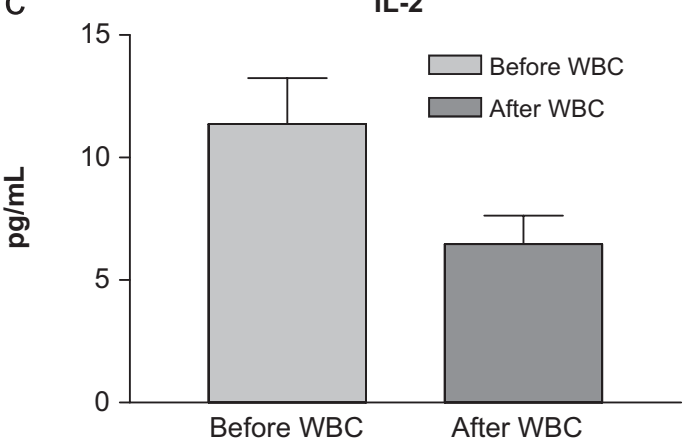

b
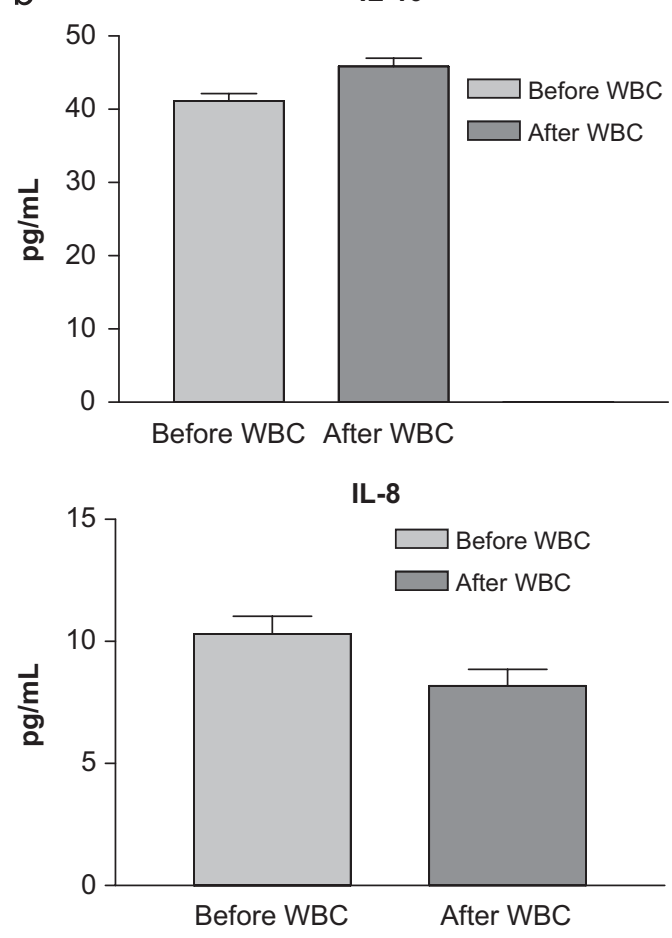

CK

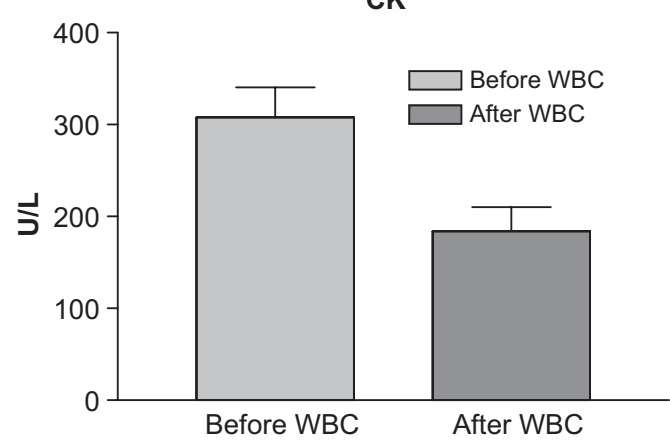

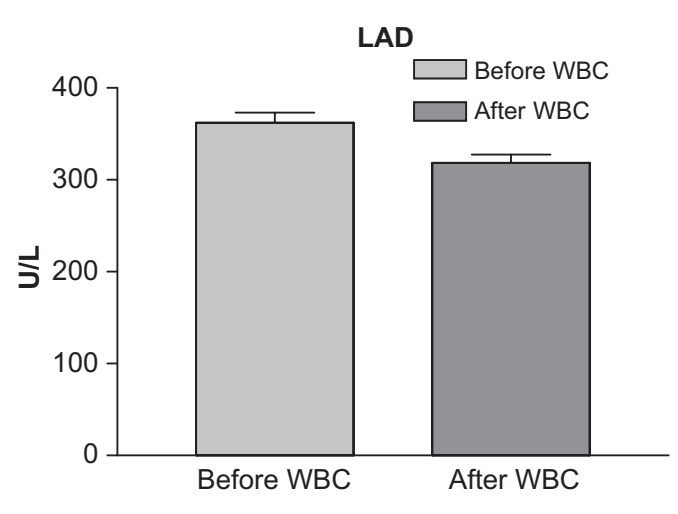

Fig. 1.

because of long duration exercise in a cold environment by experienced subjects. WBC is not characterized by changes in immunological markers and does not seem to impair immune function, as measured by using immunological parameters with paracrine activity (Dugué and Leppänen, 2000). It is not clear how WBC relieves pain and other symptoms of rheumatoid arthritis and arthropathies in general, but local cryotherapy has been shown to exert an analgesic effect on and a protective effect against collagenase on cartilage (Harris and McCroskery, 1974). The effect of WBC may be linked to alterations of paracrine molecules rather than to systemic immune functions. In sports medicine, WBC has gained wider acceptance as a procedure to improve recovery from muscular trauma; however, controlled studies on athletes are lacking. 
Published data suggest that WBC has no detrimental effect on immunological parameters, although the observation period in this study was too short to evaluate changes in lymphocyte involvement and function. Long-term cold water immersion of healthy males is known to produce slight increases in plasma TNF $\alpha$, lymphocytes and monocytes (Jansky et al., 1996) Specific studies on immunoglobulins during and after WBC are lacking, however.

Several studies have investigated the effect of cold stress on immune system function. Cold exposure in a climate chamber at $5{ }^{\circ} \mathrm{C}$ induced a small, but significant leukocytosis due to an increase in circulating neutrophils and lymphocytes, accompanied by natural killer cell activity (Brenner et al., 1999). The authors remarked that cold exposure had an immunostimulating effect that was possibly related to an enhanced noradrenaline response to the cold.

There is, in general, limited evidence indicating that short- or long-term cold exposure causes immunosuppression. In contrast, a stimulating effect of cold exposure was found to depend on the relationship between the decrease in core temperature and the duration of cold exposure (Walsh and Whitham, 2006) In one study, IgA and IgM concentrations were reported to be lower during the first 4 months of a 1-year Antarctic expedition (Gleeson et al., 2000). The change in mucosal immunity was related to the psychological stress of the expedition, but no increase in upper respiratory tract infections was observed. It could be argued that long-term cold exposure, especially when associated with psychological discomfort and mood modifications, depresses immunoglobulin production and release, whereas very short cold exposure does not affect Ig levels. Moreover, cold inhibits the expression of inflammatory mediators; hypothermia inhibits activation of neutrophils and expression of intercellular adhesion molecule-1 (ICAM-1), the adhesion and activation of neutrophils during inflammation (Hanusch et al., 2007). Therefore hypothermia induced expression of the anti-inflammatory cytokines IL-10 (Scumpia et al., 2004); hypothermia attenuates the inflammatory response during WBC, thus contributing to its beneficial role in organ protection (Hofstetter et al., 2007). As elevated serum $\mathrm{CK}$ is a characteristic marker of exertional rhabdomyolysis, it may be used for measuring the effects of workload, recovery and possible overtraining. In a previous study on top-level rugby players, we found that active recovery with leg immersion in cold water after training produced beneficial effects and a decrease in serum total CK concentration in comparison with passive recovery (Banfi et al., 2007). These results were in line with those of Gill et al. (2006) observed in rugby players by measuring CK in interstitial muscular fluid. It seems that acute exposure of the whole body to cold air stimulates muscle fiber repair by reducing cell membrane breakdown or increased cell permeability caused by oxidant agents produced during physical exercise (Banfi et al., 2006). In our subjects, the significant decrease in serum total CK concentration suggested rapid recovery from muscle damage, since the athletes did not change the training scheme or workload during the study period.

No published data are available about the behavior of $\mathrm{CK}$ and LAD after WBC. The mechanism underlying the decrease in muscle enzyme levels is unknown. The normal response of the human body to cold exposure is accelerated elimination of triiodothyronine (T3) and activation of the sympathetic nerve system, which increases the release of noradrenaline in the blood (Leppäluoto et al., 2005). The augmented T3 catabolism is not accompanied by activation of the pituitary-thyroid axis during long-term cold exposure, but rather by a hypothyroid-like status (Leppäluoto et al., 2005). Concentrations of thyroid-stimulating hormone, tetraiodothyronine and T3 were unchanged in WBCtreated patients with rheumatoid arthritis (Zagrobelny et al.,
1992). However, even if short-term cold exposure could influence thyroid metabolism, CK is elevated in subclinical hypothyroidism (Hekimsoy and Oktem, 2005). Thyroid response to cold exposure could perhaps act through a decreased sensitivity of mitochondria to ADP and creatine, as well as mitochondrial $\mathrm{CK}$, influencing the entire CK (and muscle isoenzyme LAD) metabolism (Athéa et al., 2007). Sustained noradrenaline stimulation during long-term cold exposure and WBC could play a role in relieving pain and creating an enhanced sense of well-being. However, in top-level athletes exercising in a cold environment, there is a simultaneous increase in noradrenaline and CK levels, with the noradrenaline increase generally higher than that of CK (seven-fold versus two-fold), when compared with baseline values (Rønsen et al., 2004) Noradrenaline stimulation cannot completely explain the CK decrease in our subjects after WBC treatment, but it may have triggered a cascade of events that induced the decrease.

WBC had no adverse effect on the immunological status of the subjects and had a positive effect on CK and LAD levels.

Moreover, there is an increase of anti-inflammatory cytokine IL-10, and a decrese of pro-inflammatory cytokine IL-2 and chemokine IL-8. We confirmed the decrease of sICAM-1 induced by cold treatment, inducing an anti-inflammatory response, also correlated to decrease of PGE2. PGE2 is synthesized in substantial amounts at sites of inflammation where it acts as a potent vasodilator and sinergistically with other mediators such as histamine and bradykinin causes an increase in vascular permeability and edema. Moreover, PGE2 is a central mediator of febrile response triggered by the inflammatory process and intradermal PGE2 is hyperalgesic in the peripheral nervous system.

The blood chemistry values demonstrate that this treatment cannot be considered as an illegal or unethical procedure, e.g., blood boosting (Banfi et al., 2008). Additional studies on physically active subjects are needed to confirm these findings, possibly including case-control protocol.

\section{References}

Athéa, Y., Garnier, A., Fortin, D., Bahi, L., Veksler, V., Ventura-Clapier, R., 2007. Mitochondrial and energetic cardiac phenotype in hypothyroid rat: relevance to heart failure. Pflügers Arch. 455, 431-442.

Banfi, G., Krajewska, M., Melegati, G., Patacchini, M., 2008. Effects of whole-body cryotherapy on haematological values in athletes. Br. J. Sports. Med., in press, doi:10.1136/bjsm.2007.043356.

Banfi, G., Malavazos, A., Iorio, E., Dolci, A., Doneda, L., Verna, R., et al., 2006. Plasma oxidative stress biomarkers, nitric oxide and heat shock protein in trained elite soccer players. Eur. J. Appl. Physiol. 96, 483-486.

Banfi, G., Melegati, G., Valentini, P., 2007. Effects of cold-water immersion of legs after training session on serum creatine kinase concentration in rugby players. Br. J. Sports Med. 41, 339.

Brenner, I., Castellani, J., Gabaree, C., Young, A., Zameckik, J., Shephard, R., Shek, P.N., 1999. Immune changes in humans during cold exposure: effects of prior heating and exercise. J. Appl. Physiol. 87, 699-710.

Dugué, B., Leppänen, E., 2000. Adaptation related to cytokines in man: effects of regular swimming in ice-cold water. Clin. Physiol. 20, 114-121.

Dugué, B., Smolander, J., Westerlund, T., Oksa, J., Nieminen, R., Moilanen, E., et al., 2005. Acute and long-term effects of winter swimming and whole-body cryotherapy on plasma antioxidative capacity in healthy women. Scand. J. Clin. Lab. Invest. 65, 395-402.

Fricke, L., Fricke, R., Wiegelmann, W., 1988. Beeinflussung hormoneller Reaktionen durch Ganzkorperkältetherapie. Z. Phys. Med. Baln. Med. Klim. 17, 363-364.

Gill, N.D., Beaven, C.M., Cook, C., 2006. Effectiveness of post-match recovery strategies in rugby players. Br. J. Sports Med. 40, 260-263.

Gleeson, M., Francis, J.L., Lugg, D.J., Clancy, R.L., Ayton, J.M., Reynolds, J.A., et al., 2000. One year in Antarctica: mucosal immunity in three Australian stations. Immunol. Cell. Biol. 78, 616-622.

Hanusch, C., Novak, K., Gill, I.S., et al., 2007. Hypothermic preservation of lung allograft inhibits cytokine-induced chemoatractant-1, endothelial leucocyte adhesion molecule, vascular cell adhesion molecule- 1 and intracellular adhesion molecule-1 expression. Clin. Exp. Immunol. 149, 364-371.

Harris, E., McCroskery, P., 1974. The influence of temperature and fibril stability on degradation of cartilage by rheumatoid synovial collagenase. N. Engl. J. Med. 290, 1-6.

Hekimsoy, Z., Oktem, I.K., 2005. Serum creatine kinase levels in overt and subclinical hypothyroidism. Endocr. Res. 31, 171-175. 
Hofstetter, C., Boost, K.A., Flondor, M., et al., 2007. Anti-inflammatory effects of sevoflurane and mild hypothermia in endotoxemic rats. Acta Anae. Scand. 51, 893-899.

Jansky, L., Pospisilová, D., Honzová, H., Ulicny, B., Srámek, P., Zeman, V., et al., 1996 Immune system of cold-exposed and cold-adapted humans. Eur. J. Appl. Physiol. 72, 445-450.

Leppäluoto, J., Pääkkönen, T., Korhonen, I., Hassi, J., 2005. Pituitary and autonomic responses to cold exposures in man. Acta Physiol. Scand. 184, 255-264.

Rønsen, O., Børsheim, E., Bahr, R., Klarlund, Pedersen, B., Haug, E., Kjeldsen-Kragh, J., et al., 2004. Immunoendocrine and metabolic responses to long distance ski racing in world-class male and female cross-country skiers. Scand. J. Med. Sci. Sports $14,39-48$.

Scumpia, P.O., Sarcia, J.P., Kelly, K.M., DeMarco, V.G., Skimming, J.W., 2004 Hypothermia induces anti-inflammatory cytokines and inhibits nitric oxide and myeloperoxidase-mediated damage in the hearts of endotoxemic rats. Chest 125, 1483-1491.

Smolander, J., Westerlund, T. Uusitalo, A., Dugué, B., Oksa, J., Mikkelson, M., 2006. Lung function after acute and repeated exposures to extremely cold air $(-110$ degrees $C)$ during whole-body cryotherapy. Clin. Physiol. Funct. Imaging 26, 232-234.

Walsh, N.P., Whitham, M., 2006. Exercising in environmental extremes. A greater threat to immune function? Sports Med. 36, 941-976.

Westerlund, T., Smolander, J., Uusitalo-Koskinen, A., Mikkelsson, M., 2004. The blood pressure responses to an acute and long-term whole-body cryotherapy $\left(110^{\circ} \mathrm{C}\right)$ in men and women. J. Therm. Biol. 29, 285-290.

Zagrobelny, Z., Halawa, B., Negrusz-Kawecka, M., Spring, A., Gregorowicz, H., Wawroska, A., et al., 1992. Hormonal and hemodynamic changes caused by whole body cooling in patients with rheumatoid arthritis. Pol. Arch. Med. Wewn. 87, 34-40. 